\title{
Testosterone therapy: Prescribing and monitoring patterns of practice in British Columbia
}

Jennifer A. Locke; Ryan Flannigan; Oliver P. Günther; Sean Skeldon;

S. Larry Goldenberg

Department of Urologic Sciences, University of British Columbia, Vancouver, BC, Canada

Funding: This study was supported by a data grant from the American Urological Association.

Acknowledgements: The authors thank Mahyar Etminan, Hao Luo, and Hamid Tavakoli for their early contributions to this work.

Cite as: Can Urol Assoc J 2020 July 27; Epub ahead of print. http://dx.doi.org/10.5489/cuaj.6586

Published online July 27, 2020

$* * *$

\section{Abstract}

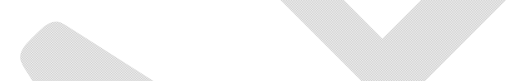

Introduction: Guidelines recommend that testosterone therapy (TTh) be restricted to men with a biochemical diagnosis of hypogonadism, and that therapeutic responses be titrated within the normal range.

Methods: Using four provincial longitudinal databases in British Columbia, we identified men prescribed TTh (1997-2013). We characterized the prescribing and monitoring practices of TTh in the context of serum testosterone levels drawn prior to and following initiation of TTh in a population-based setting.

Results: In our analysis of 37741 men who received at least one TTh prescription, 48\% received injectable testosterone and the vast majority were treated by general practitioners. The number of prescriptions for men increased annually, particularly after 2008; 40\% discontinued their treatment after their first or second prescription, while $27 \%$ received more than 10 repeats. The absolute percentage of pre- and post-serum testosterone levels ordered increased by $16 \%$ and $31 \%$ during the study period, respectively. However, after initiating TTh, only 36\% of all the men had a followup serum testosterone level drawn. Of those with low serum testosterone levels prior to TTh, $49 \%$ remained biochemically hypogonadal following TTh, suggesting non-compliance or inadequate dosing.

Conclusions: Many men prescribed TTh did not continue beyond a short trial. While the practice of checking pre-and post-TTh testosterone levels has improved over the study period, it is concerning that only one-third had a followup serum testosterone level, and 
half remained biochemically low. Further education is required around TTh prescribing, dose titration, and monitoring to ensure both effective and safe prescribing practice.

\section{Introduction}

Testosterone therapy (TTh) is approved for the management of symptomatic hypogonadism. When compared to placebo, TTh is associated with improvements in quality of life, libido, depression and erectile function as confirmed by a recent systematic review and network meta-analysis of 87 randomized clinical trials and 51 nonrandomized controlled studies ${ }^{1}$. Improved sexual functioning and quality of life compared to placebo was confirmed by a recent systematic review of 38 randomized clinical trials ${ }^{2}$. Rates of prescribing in the United States (U.S.) increased between 3 and 8-fold from 2001 to $2014^{1,3,4}$.

Coinciding with the general increased rate of TTh prescriptions over recent decades, there has been a growing concern regarding inappropriate prescribing of testosterone and lack of adherence to guidelines in both the United Kingdom (U.K.) and U.S. ${ }^{4}$. Multiple international guidelines recommend that symptomatic men may benefit from TTh only if their symptoms were associated with biochemical confirmation of hypogonadism (i.e. a total testosterone level $\leq 300 \mathrm{ng} / \mathrm{dL}(<10.4 \mathrm{nmol} / \mathrm{L})$ on 2 or more morning samples ${ }^{5-8}$ ). Despite congruent international guidelines on this, an increasing number of men may be receiving TTh despite being eugonadal, or even without any preTTh testing for a biochemical diagnosis; thus, without medical indication ${ }^{4}$. We sought to explore adherence to prescribing and monitoring practices as outlined by international guidelines in a Canadian healthcare environment that does not have the same fiscal pressures as are present in other privatized healthcare environment. We accessed, linked and analyzed four longitudinal provincial databases to address these pertinent issues.

\section{Methods}

We interrogated the population-based administrative health datasets of British Columbia (B.C.), Canada for the years 1997 to 2013, under the regulatory approval of UBC Clinical Research Ethics Board (Certificate H14-01366). British Columbia has a universal healthcare system for its 4.7 Million (as of 2015) residents ${ }^{9}$. The following databases were available: 1-Discharge Abstracts Database (DAD), containing hospitalization information including admission date and up to 25 discharge diagnoses coded using international classification of diseases, 9th (ICD-9) or 10th (ICD-10) revisions ${ }^{10}$, 2-Medical Services Plan (MSP), which contains all outpatient service dates, diagnoses, and procedural codes 11, 3-PharmaNET, which contains dispensing information such as unique drug identifiers, service date, dispensed quantities and days of supply, and medication ${ }^{12}$; 4 - outpatient laboratory results. 
The PharmaNET data was used to identify men with a first testosterone prescription during the study period, and to extract their age and respective prescriber type. A set of exclusion criteria were applied based on ICD, Hospital and MSP codes, to exclude men with prostate cancer, Klinefelter Syndrome, testicular tumors or pituitary abnormalities. A wash-in and wash-out period of one year allowed identification of preand post-treatment tests within 365 days of TTh. This period also addressed the potential problem of a man being included in the first year (1997) of our analysis as a first time TTh when in fact, it might be a repeat TTh for him.

Labvision and Sunquest datasets were combined with the LifeLabs data and when multiple rows were found in the combined testosterone lab datasets with the same lab test descriptor, collection date, test value, unit and reference range, only one of the rows was kept. Distribution of test labels and same-day co-occurring tests were tabulated and used to check for consistency.

To address the challenge of possible missing tests in the lab test datasets, the MSP data was used to identify testosterone tests based on billing codes 92266 (Total Testosterone) and 92265 (until 2011), and 92267 (2011 onwards). Similar to the lab test data, the distribution of MSP billing codes and same-day co-occurrences were tabulated and used to check for consistency. The MSP data was used to determine how many preand post-treatment testosterone tests were performed, and thus to estimate the proportion of missing results in the lab test data from PopDataBC. For the analysis, it was assumed that relative proportions of lab test result categories were representative of the proportions that would be observed in the complete dataset if it were available.

The cumulative rate of pre- or post-treatment testosterone tests was determined by dividing the number of men with a pre- or post-treatment test by the overall number of men with first TTh. A one-year time interval was used to relate tests to TTh and rates were determined over the period 1998-2013 for pre- and 1997-2012 for post-treatment tests.

PharmaNET data was used to determine prescription types (drug formulation) for first TTh. Number of first TTh, prescriber type, prescription type and total testosterone lab test value categories (when available) were aggregated by year and corresponding counts or percentages were plotted to display trends and patterns over time. Histogram displays were created to illustrate the age distribution at first TTh and for the difference in number of days of closest testosterone tests pre- and post-treatment.

Trend analyses were performed with linear regression. Residual plots and $95 \%$ confidence intervals were checked before significance of a trend was reported. 


\section{Results}

The data provided by PopDataBC identified 40,878 men who received TTh in B.C. between January 1, 1997 and December 31, 2013; after exclusion criteria were applied 37,741 men represented the TTh cohort studied in subsequent analyses.

\section{Characteristics of patients receiving first TTh prescription}

$14,889(39.5 \%)$ of the men received only $1(26 \%)$ or $2(13.5 \%)$ prescriptions suggesting a short trial of therapy with subsequent discontinuation of drug. 3,217 men (8.5\%) received $3,9,460(25.0 \%)$ men received 4-10 and 10,175 (27.0\%) men received more than 10 testosterone prescriptions over the 17-year period.

There was a 1.57-fold increase in prescriptions overall from 1998-2013 with a transient increase between 2001 and 2004, and a rising trend from 2008 onwards in men under age 80 (Figure 1a). In terms of percentage change for each of the age groups over the study period, age groups 51-60 and 71-80 showed small but significant trends with percentage changes of $0.38 \%(95 \%$ CI $[0.16,0.60])$ and $-0.28 \%(95 \%$ CI $[-0.35,-0.21])$ per year, respectively. Age group 61-70 had a percentage increase of $0.17 \%$ (95\% CI $[0.02,0.33])$ per year while age groups $40-50,81-90$ and $>90$ displayed small, nonsignificant changes (Figure 1b). The youngest and oldest ages at first TTh prescription were 28 and 109 years old, and the mean and median ages at first TTh were 60 and 59 years (Figure 1c). Standard deviation and median absolute deviation were 11 years.

\section{TTh prescription types}

Overall, the majority of prescription types were injectables (48\%), followed by oral capsules (25\%), gels (24\%) and patches (3\%) (Figure 2). Prior to 2001 only two treatments were available: injectables and capsules. From 1998-2000 there was a significant decrease in injectables, $-3.36 \%$ per year (95\% CI [-3.88, -2.83]) and a significant increase in capsules, $3.36 \%$ per year (95\% CI [2.83, 3.88]). From 2001-2004, with the introduction of other formulations, both the injectable and capsule prescriptions decreased as the newer formulations increased. The proportionate use of gel prescriptions increased steadily from $2005(26 \%)$ to 2013 (43\%) with an annual increase of $2.29 \%$ $(95 \%$ CI $[1.83,2.76])$. During these years linear downward trends were found to be significant for capsules $(-2.28 \%, 95 \%$ CI $[-2.60,-1.96])$ and patches $(-0.48 \%, 95 \%$ CI [$0.64,-0.32])$.

The median durations of TTh formulations were 68 months for injectables, 90 months for capsule, 90 months for gel and 60 months for patch.

\section{Patterns in serum testosterone testing}

Initial serum testosterone measurements are used to provide a biochemical diagnosis supporting clinical hypogonadism; this is considered a standard of care ${ }^{5-8}$. Post-TTh serum testosterone measurements are recommended to ensure appropriate dose titration 
of $\mathrm{TTh}^{5-8}$. During this study period, the percentage of men in whom serum testosterone tests (either total or free) were drawn within 1 year prior to their first prescription rose from $69 \%$ in 1998 to $85 \%$ in 2013 (Figure 3a). This represented a significant linear trend with a percentage increase of $1.27 \%(95 \%$ CI $[1.06,1.48])$ per year. The percentages of post prescription measurements rose from $20 \%$ in 1997 to $51 \%$ in 2012 , for a cumulative rate of post-TTh testing of $36 \%$ (12,539 of 34,582 men overall). The observed linear trend was significant with a percentage increase of $2.09 \%$ (95\% CI $[1.85,2.34])$ per year. The majority of men started TTh treatment soon after being tested (median duration, defined as date of closest pre-treatment serum testosterone test to date of first TTh $=19$ days) (Figure 3b); while there was a longer amount of time for the post-serum testosterone draw (median duration, defined as date of first TTh to closest post-treatment serum testosterone test $=87$ days).

Percentages of total testosterone measurements for men with matching same-day MSP codes were categorized as low ( $<9 \mathrm{nmol} / \mathrm{L}$ ), borderline ( 9 to $<12 \mathrm{nmol} / \mathrm{L}$ ) or normal $(12-28 \mathrm{nmol} / \mathrm{L})$ (Figure 4). Between 2009 and 2010 there was a significant increase in the percentage of low pre-treatment tests and an accompanying decrease in normal and borderline pre-treatment tests.

For those men who had low pre-TTh serum testosterone measurements $49 \%$ remained low post-TTh and $51 \%$ became borderline or normal/high post-TTh (Table 1). Curiously, $26 \%$ of men with borderline and $11 \%$ with normal testosterone at baseline actually had low levels after starting therapy.

\section{Prescribing physicians}

From 2008 to 2013 there were increasing counts of TTh prescriptions; mostly written by general practitioners, followed by urologists, internal medicine physicians and endocrinologists (Table 2).

In terms of percentage of physician prescriber type ordering a pre-TTh serum testosterone test, general practitioners displayed a significant percentage-increase of $1.39 \%$ per year, $95 \%$ CI $[1.17,1.61]$ over the course of the study, while increases for urology and internal medicine were $0.47 \%, 95 \%$ CI $[0,0.93)$ and $0.93 \%, 95 \%$ CI [0.06, 1.81]. Trends for endocrinologists were not significant (Figure 5a). For post-TTh serum testosterone tests, percentage increases for general practitioners $(2.01 \%, 95 \%$ CI $[1.75$, 2.27 ] urology $(3.26 \%, 95 \%$ CI [2.82, 3.69]) and internal medicine $(2.11 \%, 95 \%$ CI [1.47, 2.76]) were significant. General practitioners consistently ordered fewer labs as compared to urologists, internal medicine and endocrinologists (Figure 5b). The median duration of prescription was longest when prescribed by endocrinologists (120 months) and shortest when prescribed by general practitioners (70 months) (Table 2$)$. 


\section{Discussion}

Our analysis of 37,741 men revealed that the number of men prescribed testosterone in B.C. has increased 1.57-fold between 1998-2013, with a steeper increase in the trend after 2008. The magnitude of trend from 2008 onwards was similar to that observed in the U.S. over a similar time period (3-8 fold) ${ }^{1,3,4}$, and may be related to the availability of transdermal testosterone formulations (i.e. gel and patch).

Injectables were the most commonly prescribed form of testosterone replacement therapy from 1998-2013; however, there was an expected rise in gels when they came to market. We note that most recent American College of Physicians guidelines favor the use of intramuscular rather than transdermal formulations to improve sexual function at a lower cost with similar clinical effectiveness and potential harms ${ }^{8}$. Other international guidelines do not specify best prescription type ${ }^{5-8}$. As such, various options for TTh are available with varying strengths and weaknesses, and likely require individualized treatment approaches based upon patients' needs and preferences.

Despite median duration of therapy ranging between 70-120 months, nearly half of all men only filled 1-3 prescriptions, suggesting willingness to trial but not continue therapy. This pattern may be due to inadequate response or unwanted side effects. Investigating this further, we found that among men with low pre-TTh serum testosterone levels, half remained low following TTh, while only $37 \%$ obtained a normal serum testosterone level. Furthermore, 37\% of men with borderline or normal serum testosterone levels pre-TTh were found to have low levels post-TTh. This may be due to the timing of lab testing occurring during a trough level; or, to the exogenous dosing negatively regulating pituitary production of $\mathrm{LH}$, through the classically described negative feedback mechanism ${ }^{13}$. This would result in minimal testicular testosterone production while exogenous doses were inadequate to fully support eugonadal levels. Other investigators have not found a correlation between the relief of hypogonadal symptoms and pre-TTh serum testosterone levels ${ }^{14}$ emphasizing the requirement for proper dose titration guided by serum levels post-TTh ${ }^{5-8}$. Further studies are required to better optimize the timing of post-TTh serum testosterone testing (peak, trough or mid cycle).

Not surprisingly, the most common prescribers of TTh in Canada are general practitioners, followed by urologists and internists. Our analysis showed comparable prescribing patterns of practice between the various physician groups. Although Canadian general practitioners were the major drivers of the overall increase in the number of serum testosterone measurements taken prior to prescribing testosterone (1998-2013) the situation differed for post-prescription serum testosterone levels as the number of general practitioners requesting this measurement did not increase in proportion to other prescribers, particularly urologists (1997-2012). Furthermore, median duration of prescription was shortest when prescribed by general practitioners. Improving 
education of general practitioners on the importance of ordering post-TTh serum testosterone levels as well as optimal dose titration methods may help address the observed low compliance of TTh.

A limitation of our study was the challenge of accessing all of the actual serum testosterone measurement values from data provided by PopDataBC. However, the data that documented ordering of a serum testosterone test was complete, as it was completely captured in the MSP database. This allowed for an albeit smaller, but accurate subgroup analysis to evaluate the measured testosterone levels. Also, measurement of serum testosterone values following TTh is complicated by the variability due to timing relative to dose administration (i.e. trough, peak, mid-cycle) in men on injectable drugs and we chose to focus on the first testosterone measurement following TTh prescription; thus, it is possible that with subsequent and different timing of testing, testosterone values may have responded differently over time. Lastly, in our databases we do not have access to patients' hypogonadal symptoms (presence and degree), and so could not assess clinical response to treatment. Lastly, we cannot determine with certainty whether lack of postTTh testing was due to physician practice or patient compliance.

\section{Conclusions}

Overall, the results of this study highlight significant deviations from standard-of-care for TTh prescribing in Canada. In particular, nearly half of the men do not remain on TTh for more than a short trial period. Adherence to guidelines on suggested monitoring of serum testosterone levels before and after initiation of TTh has improved, but rates remain unacceptably low with only $36 \%$ of all the men receiving post-TTh serum T monitoring. Among those being tested, approximately half of men with low testosterone prior to TTh, remain low following therapy. We suggest that the absence of biochemical testosterone assessment both before and after treatment likely contributes to inappropriate prescribing, poor adherence and inadequate therapeutic response. As a group, general practitioners were the least adherent to ordering post-TTh serum testosterone levels and thus may benefit from future directed educational initiatives. Further studies must also address the issue of optimizing the timing of serum testosterone level measurements; considering we found significant variations in pre- and post-TTh serum testosterone levels. Overall, clinician education is required around testosterone therapy prescribing, dose titration and monitoring to ensure both effective and safe prescribing practice. 


\section{References}

1. Elliott J, Kelly SE, Millar AC et al: Testosterone therapy in hypogonadal men: a systematic review and network meta-analysis. BMJ Open 2017; 7: e015284.

2. Diem SJ, Greer NL, MacDonald R et al: Efficacy and Safety of Testosterone Treatment in Men: An Evidence Report for a Clinical Practice Guideline by the American College of Physicians. Ann Intern Med 2020; .

3. Baillargeon J, Kuo YF, Westra JR et al: Testosterone Prescribing in the United States, 2002-2016. JAMA 2018; 320: 200.

4. Layton JB, Li D, Meier CR et al: Testosterone lab testing and initiation in the United Kingdom and the United States, 2000 to 2011. J Clin Endocrinol Metab 2014; 99: 835 .

5. Morales A, Bebb RA, Manjoo $P$ et al: Diagnosis and management of testosterone deficiency syndrome in men: clinical practice guideline. CMAJ 2015; 187: 1369.

6. Mulhall JP, Trost LW, Brannigan RE et al: Evaluation and Management of Testosterone Deficiency: AUA Guideline. J Urol 2018; 200: 423.

7. Lunenfeld B, Mskhalaya G, Zitzmann M et al: Recommendations on the diagnosis, treatment and monitoring of hypogonadism in men. Aging Male 2015; 18: 5 .

8. Qaseem A, Horwitch CA, Vijan S et al: Testosterone Treatment in Adult Men With Age-Related Low Testosterone: A Clinical Guideline From the American College of Physicians. Ann Intern Med 2020; .

9. Central stats agency: Population Estimates,BC Stats,central statistical agency of the Province of British Columbia 2015;

10. Discharge Abstract Database (Hospital Separations). Population Data BC: Canadian Institute for Health Information 2014; .

11. British Columbia: Ministry of Health Services MSP fee-for-service payment analysis | BC Government Publication | Legislative Library | Legislative Assembly of British Columbia 2010; .

12. PharmaNet: BC Ministry of Health [publisher]. Data Extract. Data Stewardship Committee 2013; .

13. Bagatell CJ, Dahl KD, Bremner WJ: The direct pituitary effect of testosterone to inhibit gonadotropin secretion in men is partially mediated by aromatization to estradiol. J Androl 1994; 15: 15.

14. DeFina LF, Radford NB, Leonard D et al: Traditional signs and symptoms commonly attributed to hypogonadism do not correlate with testosterone levels: the Cooper Center Longitudinal Study Experience. J Investig Med 2019. 
Figures and Tables

Fig. 1. Annual distribution of (A) first testosterone therapy prescription counts and (B) percentage for 37741 men from 1997-2013, stratified by age at first treatment. *576 men younger than 40 years of age at time of first treatment were excluded in Fig. 1A.
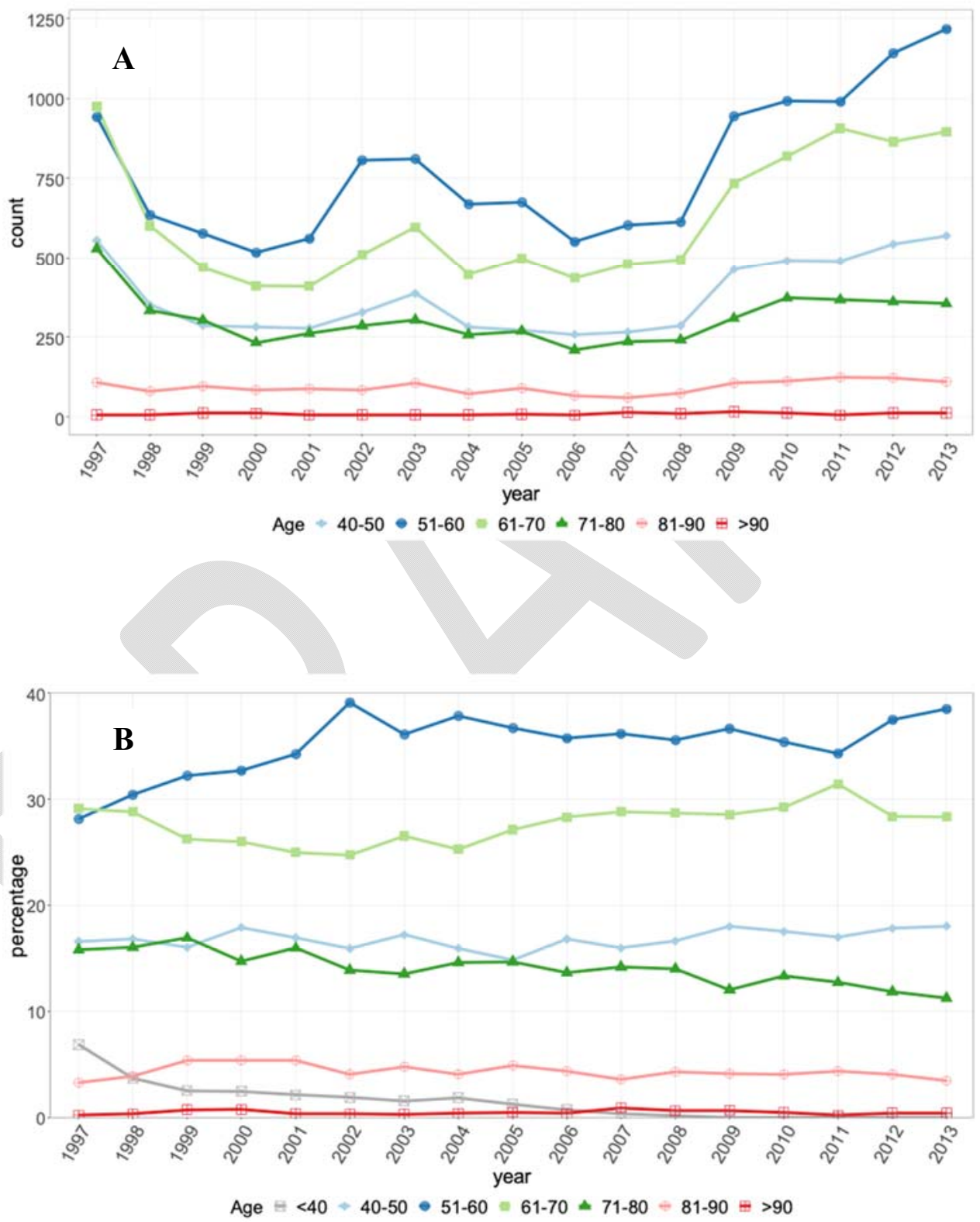
Fig. 1C. Distribution of first testosterone therapy prescription by age (n=37 $741 \mathrm{men}$ ).

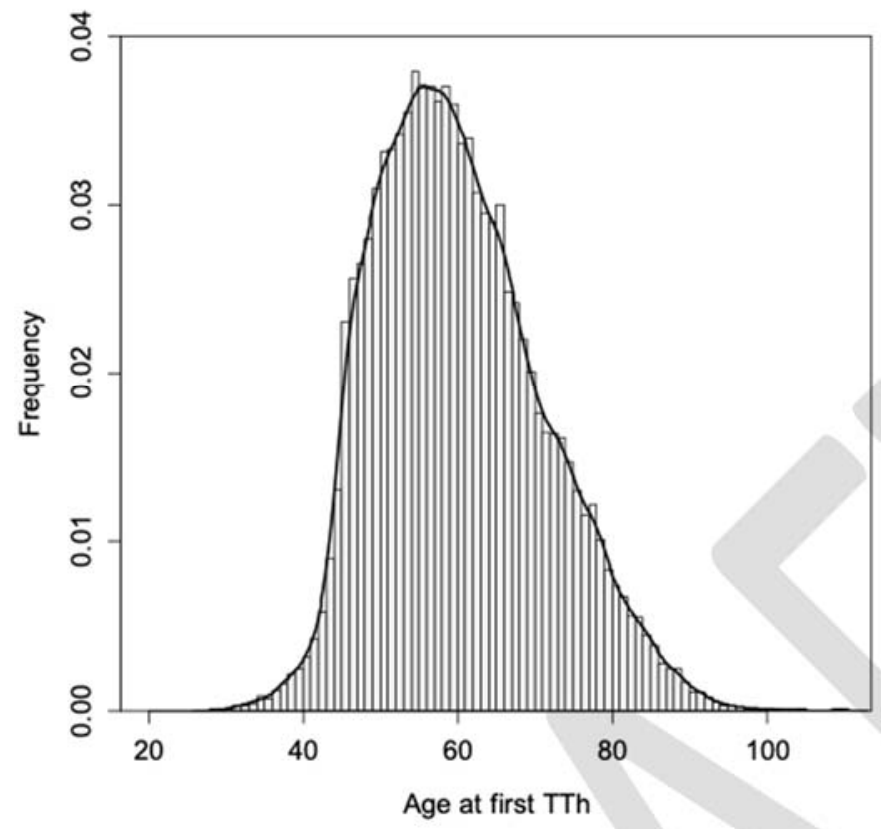

Fig. 2. Distribution of treatment types at first testosterone therapy by year. "Prior to 2001, there were only two treatment types used (injectables and capsule), after which gel and patch formulas became options for patients.

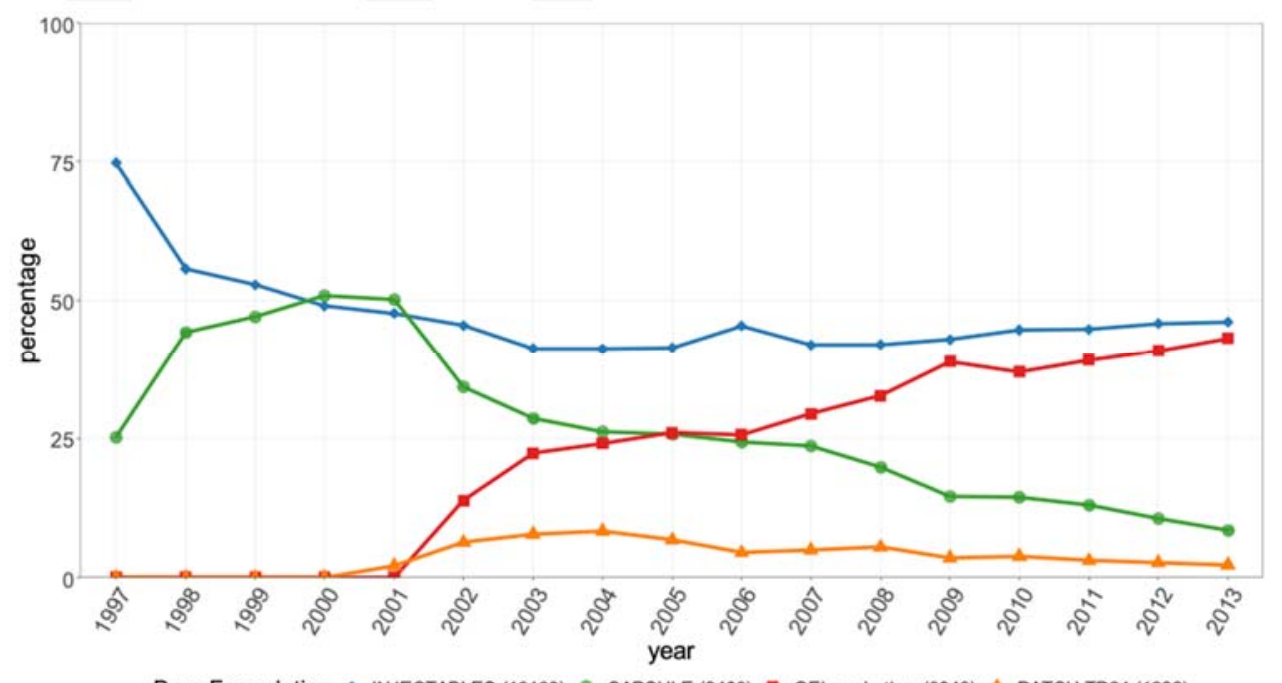

Drug Formulation + INJECTABLES (18189) - CAPSULE (9408) - GEL and other (8943) - PATCH TD24 (1296) 
Fig. 3A. Percentages of pre- and post-testosterone therapy serum testosterone tests performed within a one-year time-window of first prescription. "The pre-treatment test curve starts in 1998 to allow capture of pre-treatment tests in the previous year. The posttreatment test curve ends in 2012 to allow capture of post-treatment tests in the last year of the study period.

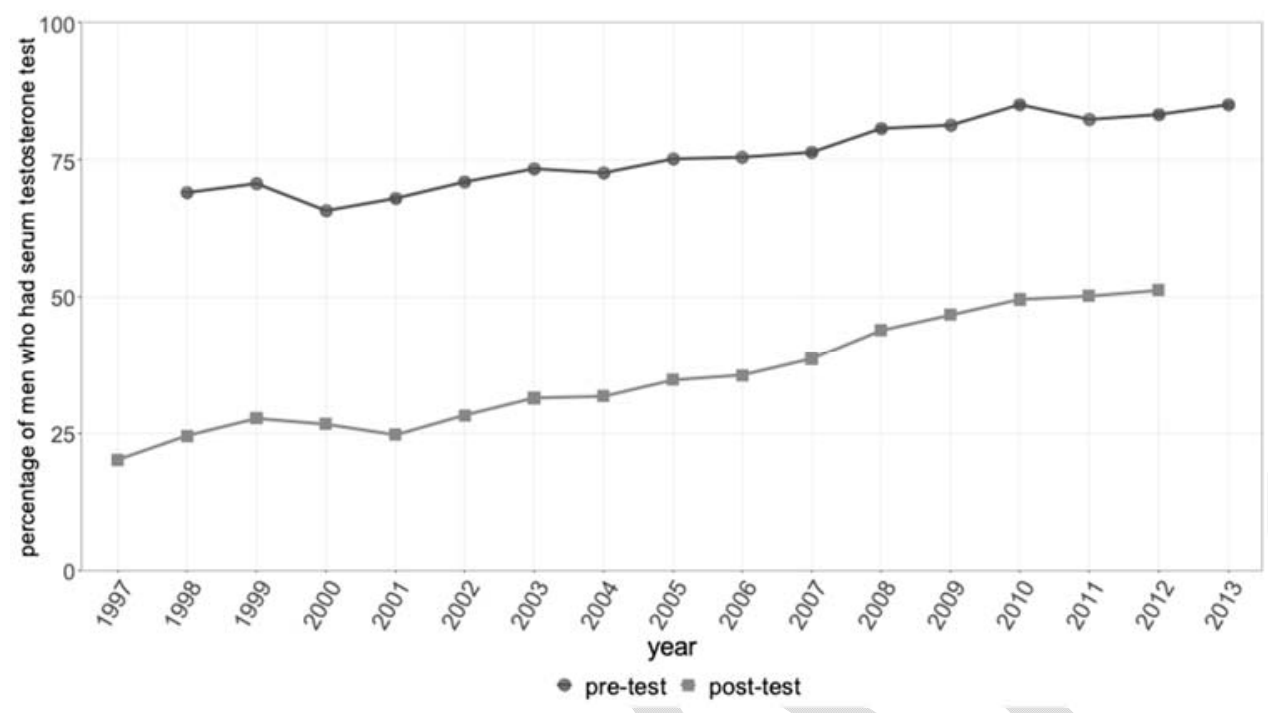

Fig. 3B. Distribution of the time interval of pre- and post-serum testosterone measurement relative to the date of first testosterone therapy (TTh) prescription within a one-year time window.

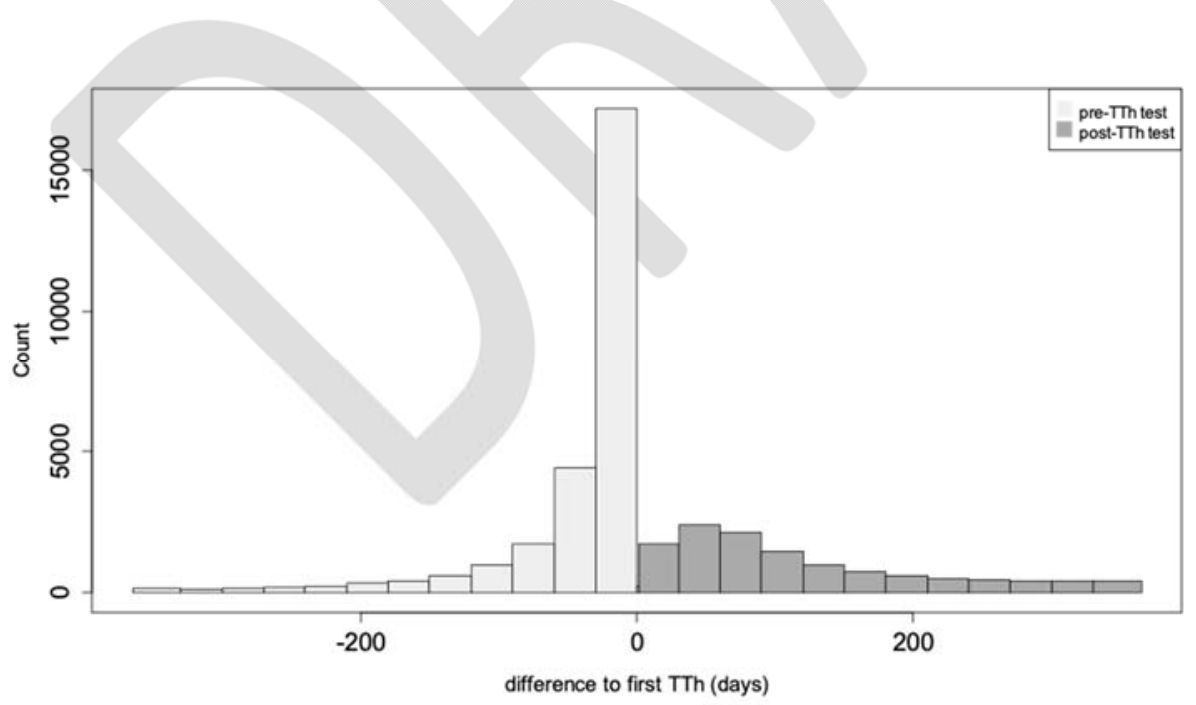


Fig. 4. Percentage of total testosterone measurements for men with matching same-day MSP codes were categorized as low $(<9 \mathrm{nmol} / \mathrm{L})$, borderline $(9$ to $<12 \mathrm{nmol} / \mathrm{L}$ ) or normal $(12-28 \mathrm{nmol} / \mathrm{L})$.

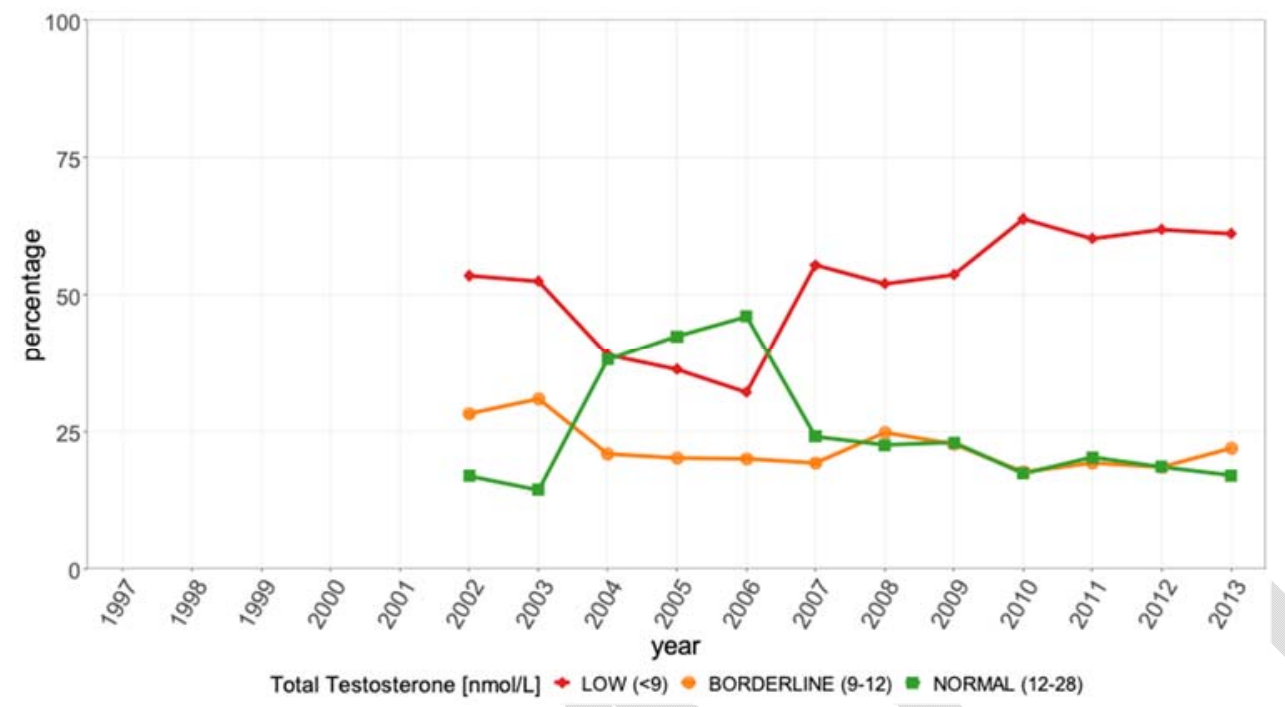

Fig. 5A. Percentage of each practitioner type that are ordering a pre-testosterone therapy (TTh) serum total testosterone measurement ( $\mathrm{n}=26083$ first $\mathrm{TTh}$ ) as analyzed on an annual basis from 1998-2013.

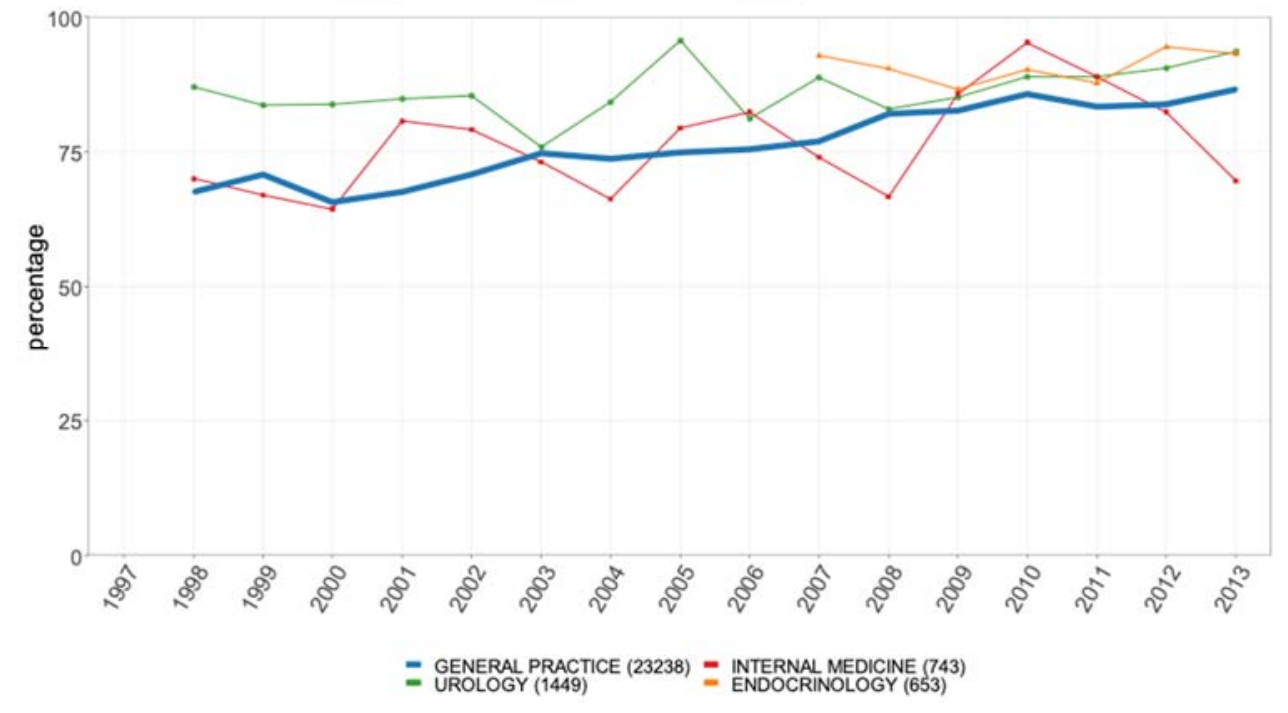


Fig. 5B. Percentage of each practitioner type that are ordering a post-testosterone therapy (TTh) serum total testosterone measurement ( $\mathrm{n}=12276$ first TTh) as analyzed on an annual basis from 1997-2012.

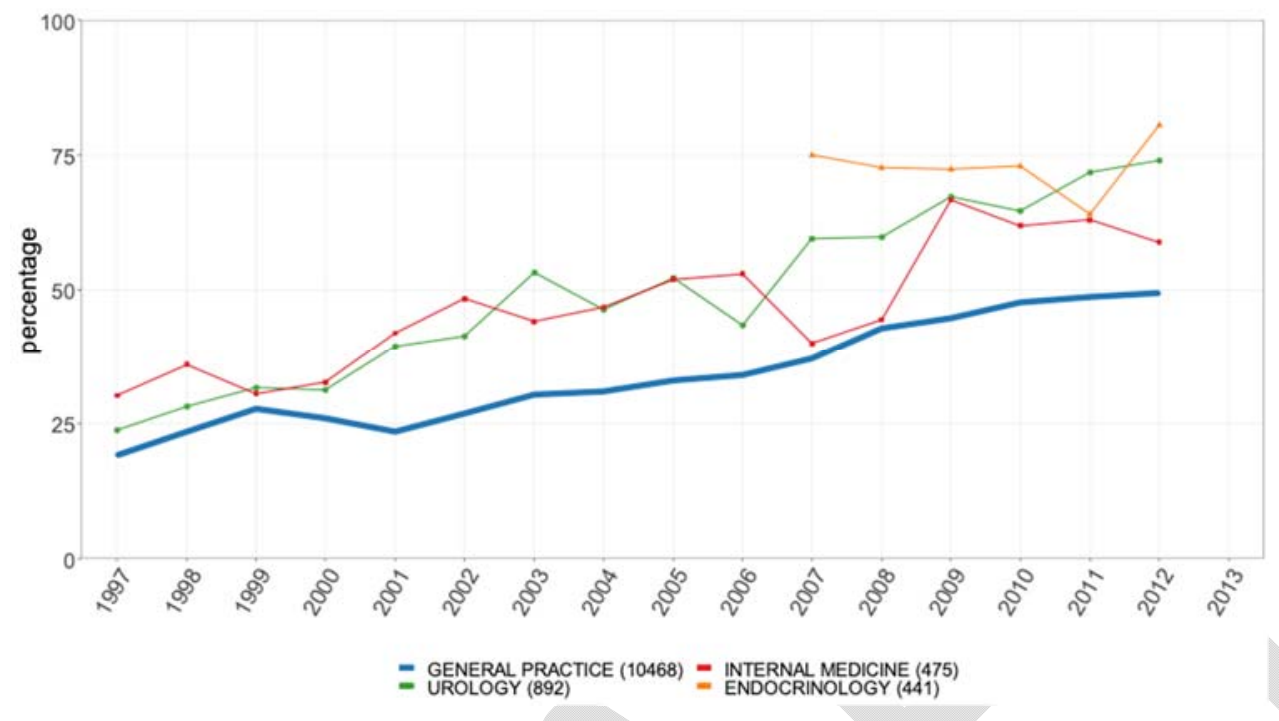

Table 1. Summary of absolute counts and percentages of men with low, borderline normal and high serum total testosterone levels pre-TTh (rows) and post-TTh (columns) testing

\begin{tabular}{|l|c|c|c|c|c|}
\hline & $\begin{array}{c}\text { Low } \\
\text { (post) }\end{array}$ & $\begin{array}{c}\text { Borderline } \\
\text { (post) }\end{array}$ & $\begin{array}{c}\text { Normal } \\
\text { (post) }\end{array}$ & High (post) & $\begin{array}{c}\text { Total } \\
\text { count }\end{array}$ \\
\hline Low (pre) & $263(49 \%)$ & $76(14 \%)$ & $163(30 \%)$ & $40(7 \%)$ & 542 \\
\hline Borderline (pre) & $39(26 \%)$ & $31(21 \%)$ & $61(41 \%)$ & $19(13 \%)$ & 150 \\
\hline Normal (pre) & $14(11 \%)$ & $22(17 \%)$ & $81(61 \%)$ & $15(11 \%)$ & 132 \\
\hline
\end{tabular}

There were only six men with a total testosterone pre-TTh level in the high category and they were excluded in the table. TTh: testosterone therapy.

\begin{tabular}{|l|c|c|c|c|c|}
\hline \multicolumn{6}{|c|}{ Table 2. Distribution of prescriber types by median duration of TTh prescription } \\
\hline Prescriber type & $\begin{array}{c}\text { General } \\
\text { practice }\end{array}$ & Urology & $\begin{array}{c}\text { Internal } \\
\text { medicine }\end{array}$ & Endocrinology & Other \\
\hline $\begin{array}{l}\text { Number of first } \\
\text { TTh }\end{array}$ & 32739 & 1991 & 1152 & 720 & 405 \\
\hline $\begin{array}{l}\text { Median duration } \\
\text { (months) }\end{array}$ & 70 & 90 & 101.5 & 120 & 90 \\
\hline
\end{tabular}

TTh: testosterone therapy. 